American Journal of Applied Sciences 7 (10): 1310-1316, 2010

ISSN 1546-9239

(C) 2010 Science Publications

\title{
Aluminum Powder and Zwitrionic Surfactants as Drag Reducing Agents in Pipe Lines
}

\author{
Hayder A. Abdul Bari, Rosli Bin Mohd Yunus and Tania Suhail Hadi \\ Faculty of Chemical and Natural Resources Engineering, \\ University Malaysia Pahang, Locked Bag 12, \\ 25000, Kuantan, Pahang, Malaysia
}

\begin{abstract}
Problem statement: One of the most power consuming sector in the industry is the liquid transportation through pipelines due to the turbulent mode the liquids are transported with. Drag reducing agents were used as a solution for the pumping power losses in pipelines. One of the inportant drag reducing agents suggested to improve the flow in pipelines are the suspended powders. Approach: In the present study, aluminum powders and zwitrionic surfactant ((3-(Decyldimethyleammonio) propanesulfonate inner salt and 3-(n-n Dimethylpalmityl-ammonio propanesulfonate) were investigated as drag reducing agent in aqueous media. The effect of additive concentration, Reynolds number and the testing section length are the main variables investigated. All the experimental work was carried in a build up experimental rig that consist of a closed loop experimental piping system. Results: The experimental results showed that, the percentage drag reduction $\mathrm{Dr}(\%)$ increases by increasing the additive concentration and Reynolds number with maximum percentage drag reduction up to $50 \%$ with only $500 \mathrm{ppm}$ addition concentration. Conclusion: The effect of testing section length was not so clear due to the way of introducing the additive to the main flow, that the additive is mixed in the main tank and not injected.
\end{abstract}

Key words: Drag reduction, zwitterionic surfactants, flow improving, pumping power saving

\section{INTRODUCTION}

One of the major problems facing the modern industrial development is the power resources. From the economical point of view; pumping power losses during the flow of transported liquids in pipelines are one of the major problems facing many industrial applications (especially petroleum).

The resistance that the fluid faces during its flow in pipeline is called "Drag". This resistance force is parallel to the direction of fluid flowing over a solid surface. Drag force may be expressed by two components: "Skin friction component" which is equal to the stream wise component of all shearing stresses over the surface and "pressure drag component" which is equal to the stream wise component of all normal stresses.

The addition of small amount of chemical additives to the solution in the turbulent flow has been proven to reduce the drag caused by turbulence in pipelines.

The most famous drag reducing agents are: Polymers, fibers, soaps or surfactants. Drag Reduction as a science have great economical benefit because of the high capability of energy consumption reduction (pumping energy consumption). In another words; increasing the flow in pipelines without the need for changes in the mechanical parts of the process such as the size of these pumps, pipes and fittings.

Even though the intensive researchers have been done, a fully accepted theory behind the drag reduction does not exist. The reason for the difficulty is the nature of the problem; it is a combination of physics, chemistry, rheology and hydrodynamic. The chaotic media that the drag reducer works in (turbulent flow), where masses of liquid moves randomly through the pipe in non-predictive manner and the absence of a modern technique to establish a clear mapping of turbulence inside the pipe, made all the mechanisms suggested highly speculative and all have been subjected to criticism. However, the major categorize of drag reduction mechanisms suggested in the literatures were, adsorption mechanism, structure mechanism and elastic-viscous mechanism. On the other hand, high percentages of drag reduction were established using fiber suspensions. This technique made the prediction of the drag reduction mechanism harder, because there

Corresponding Author: Hayder A. Abdul Bari, Faculty of Chemical and Natural Resources Engineering, University Malaysia Pahang, Locked Bag 12, 25000, Kuantan, Pahang, Malaysia 
is another phase presented during the turbulent flow and that minimized the idea of chemical additives dilution in transported fluid.

Generally, DRA are viscoelastic, time-independent, shear degradable, non-Newtonian fluids. DRA's in present use in oil and products pipelines are themselves hydrocarbons and thus should have no effect on refining processes or refined products. Zwitterionic surfactant is a surfactant which its head contains tow oppositely charged groups.

Indartono et al. (2005) investigated the temperature and diameter effect on hydrodynamic characteristic of cationic surfactant drag reducing flows in pipes. They made their tests at $12,25,40$ and $50^{\circ} \mathrm{C}$ in pipes with diameter of 13,25 and $40 \mathrm{~mm}$ on solution of olelylbhydroxyethyle methyl ammonium chloride (Ethoquad O/12)(900 ppm) as cationic surfactant and sodium salicylate (nasal)(540 ppm) as counter ion. DR effectiveness of this surfactant solution was evaluated in $25 \mathrm{~mm}$ pipes from $6-75^{\circ} \mathrm{C}$ Rheological characteristic of this solution was measured by stress control type rheometer with cone and plate geometry. They found in their research that this surfactant solution has clear DR capability until $70^{\circ} \mathrm{C}$ and they suggested that temperature has a significant influence in changing the hydrodynamic entrance length of surfactant drag reducing flows. And it was found that, from rheological measurements, the solution exhibits shear induced structure at all temperatures with different degree of peak viscosity and critical shear rate.

Jaghmani (1999) investigated the effect of nonionic surfactant $(\mathrm{X}-100)$ on rheological properties of polyacrylamide, using cone and plate rheometer. Polyacrylamide and surfactant in the test solution test ranged by weight from (0.02-0.1\%) and (0.001-0.05\%) respectively. The scope of the study includes the measurements of surface tension, steady state behavior, yield stress, creep and recovery, thixotropy, transient shear stress response and dynamic response.

The results have shown decrease in the surface tension with increasing surfactant up to the concentration. Polyacrylamide solutions have shown three different behaviors, the first is Newtonian region, then a region of dramatic shear thinning and finally a shear thicken region.

Dynamic viscosity have shown shear thinning up to a critical value above which the solutions gave constant shear thickening behavior and also showed that coxmerz rule is not valid in this range of tested solutions. The viscoelastic and thixotropic responses of the tested solutions were small. The effect of surfactant was low.

Zhang et al. (2005) investigates DR and Rheological properties of two cationic surfactants, Ethoquad $\mathrm{O} / 12$
(Oleylterion $\mathrm{NaSl}$ ), in mixed solvents containing 0-28 wt $\%$ Ethylene Glycol (EG) and water. The addition of EG to the solvent had grater of solutions DR ability, shear viscosity, apparent extensional viscosity and viscoelasticity at $25^{\circ} \mathrm{C}$ than at $0^{\circ} \mathrm{C}$. Cryo-TEM images show thread like micelle in these systems. DR at low temperatures' in solutions containing moderate amount of EG can be utilized in a new approach to energy saving in district cooling systems using EG-water based mixtures as he cooling fluids.

Savines (1964) observed an interesting stress controlled DR effect, in the soap solutions. DR increase with increasing shear stress up to a critical value. Beyond the critical value, the DR of the soap solution become indistinguishable from that of the soap free solution. This indicated that the network of micelles collapses if the shear stress exceeds a critical shear stress. This occurs because of a temporary disentanglements of the network include by turbulent vortices and eddies in fully development flow. If the wall shear stress is reduced from above to below the critical value, then the network bounds reform and the reducing ability of the solution is restored. In contrast the polymers chains are broken by high shear stress, the drag reducing ability of the polymer solution is permanently lost. The critical shear stress where mechanical degradation starts in dilutes polymer solutions.

Ananthapadmanabhan et al. (1991) studded the surface forces measurements between adsorbed layers on mica of cationic polymers (JR-400 polymer) in distilled water, both in absence and in the presence of the anionic surfactant sodium dodecyl sulphate. In the absence of surfactant, the polymer was bound to the oppositely charged mica surface and exhibited hysteresis force curves which were monotonically repulsive up to compression of $10^{4} \mu \mathrm{N} \mathrm{m}^{-1}$. In the presence of surfactant hysteresis was also observed but while the compressing force curves were monotonically repulsive, the separating force curves showed a significant attraction between the layers, this suggests that bound surfactant may be including hydrophobic interactions between the absorbed layers.

Chapman (2005) determined for both Zwitterionic and Non-ionic surfactant in $20 \%$ by weight ethylene glycol/water that could be used in district cooling system for the temperature range of $(-5-15)^{\circ} \mathrm{C}$ drag reduction other solvent such as water $30 \%$ by weight glycerol/water and $25 \%$ propylene glycol/water were suggested also. One intention of this project was to find more environmentally benign surfactants with drag reducing ability equal to that of cationic surfactants. Addition of sodium nitrite have been shown the 
contribute to drag reduction as well as being an effective means of preventing corrosion in the metal pipes of a circulation system when used in combination with Zwitterionic/non-ionic or Zwitterionic/anionic surfactant solutions. Finding an effective concentration of sodium nitrite to add to surfactant solutions was the goal of his study. In some cases additions anionic surfactants to Zwitterionic surfactant has enhanced effectiveness in reducing drag and therefore determining an effective Zwitterionic: Anionic ratio was another goal to his study and the objective of his study was finding an effective drag reducing system by evaluating formulations.

\section{MATERIALS AND METHODS}

Transported fluid: The transported fluid used in the present investigation was water.

\section{Liquid circulation system and experimental procedure:}

Experimental rig: An experimental rig was built in order to achieve the aims of this experimental work. The main components of the system consisted of horizontal test section and instrumentations. The pipes with $0.0127,0.0254$ and $0.0381 \mathrm{~m}$ inside diameter and the length of $2 \mathrm{~m}$ made from transparent PVC pipe to permit visual observation of flow pattern in the future was used as the test section. Each pipe divided into four pressure testing sections with a distance equal to $0.5 \mathrm{~m}$. The first pressure testing point for each pipe was located about 50 times pipe diameter $(50 \mathrm{D})$ of the testing pipe as shown in Fig. 1. This is to ensure the turbulent flows are fully developed before the testing point. As shown in Fig. 1, the smallest diameter of pipe which is pipe $\mathrm{C}$ are built as an alternative to control the flow in pipe and as a replacement for bypass pipe since bypass pipe was sometime not appropriate during the experimental work due to formation of heavy foam.

Ultraflux portable flow meter Minisonic P: The flow rate of fluid in pipelines was measures by Ultraflux Portable Flow meter Minisonic $P$ in which this ultrasonic flow measurement was sensitive with small changes in flow rate as low as $0.001 \mathrm{~m} \mathrm{sec}^{-1}$. The purpose of using this exterior portable ultrasonic measurement is to avoid the pressure losses and prevents leaking that may associate with in-line meter installation.

Baumer differential pressure gauges: Baumer Differential Pressure Gauge is used to detect the pressure drop in pipelines in which this pressure measurement capable to read the pressure difference in pipelines up to 0.16 bar. In addition, this pressure measurement has high sensitivity and the instrument installation is easy. This pressure gauge was installed in a middle of each testing section in order to make the portable tube easy to remove and connected to the pressure gauge from the testing section during the experiment work.

\section{Experimental calculations:}

Velocity and Reynolds number calculations: The average Velocity (V) and Reynolds number (Re) were calculated using the solution volumetric flow rate readings $(Q)$, density $(\rho)$, viscosity ( $\mu$ ) And pipe diameter (D), for each run as follows:

$\operatorname{Re}=\frac{\rho \cdot V \cdot D}{\mu}$

Percentage drag reduction calculations: Pressure drop readings through testing sections before and after drag reducer addition, were needed to calculate the percentage drag reduction $\operatorname{Dr}(\%)$ as follows:

$$
\operatorname{Dr}(\%)=\frac{\Delta \mathrm{P}_{\mathrm{b}}-\Delta \mathrm{P}_{\mathrm{a}}}{\Delta \mathrm{P}_{\mathrm{b}}}
$$

Where:

$\Delta \mathrm{P}=$ Pressure drop after drag reducer addition

$\Delta \mathrm{P}=$ Pressure drop before drag reducer addition

The friction factors for pure water flow and maximum drag reduction asymptotes were calculated based on equation as specified below:

$$
f=\frac{2 \tau_{w}}{\rho V^{2}}
$$

Where:

$$
\tau_{\mathrm{w}}=\frac{\mathrm{D} \Delta \mathrm{P}}{4 \mathrm{~L}}
$$

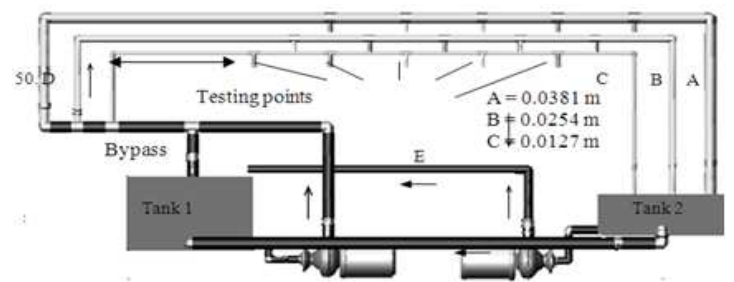

Fig. 1: The experimental rig 
The Blasius equation is defined as (Cengel and Cimbala, 2006):

$\mathrm{f}=0.0791 \mathrm{Re}^{-0.25}$

and Virk's asymptote is defined as (Virk et al., 1970):

$\mathrm{f}=0.59 \mathrm{Re}^{-0.58}$

whereas the Reynolds number in pipe is defined as:

$\operatorname{Re}=\frac{\rho \mathrm{VD}}{\mu}$

Moreover, the friction factors in pipes based on laminar flow are defined as:

$f=\frac{16}{\operatorname{Re}}$

\section{RESULTS}

Friction factor: One of the main representations for the experimental work of drag reduction and the pumping power savings occurred during the experiments is the friction factor effect with the percentage drag reduction in each experiment. Figure 1-4 shows the relation between the Dr (\%) and the friction factor $\mathrm{f}$. The experimental work showed a good logical agreement in the relation between the Dr (\%) and the friction factor with the ones mentioned in the literatures.

It can be seen clearly that the $\operatorname{Dr}(\%)$ increases with any decrease in the friction factor caused by the drag reduction effect of the additives. In this case it is clear that by the addition of Zwitterionic surfactant additive $(3 \mathrm{NN})$ to the solid-liquid flow system, the $\mathrm{Dr}(\%)$ increases more than before and the relation between the $f$ and Dr (\%) still the same (in the behavior) compared with the previous relation.

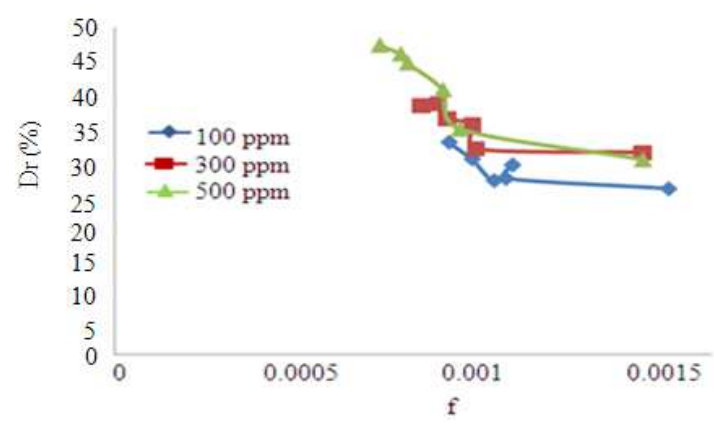

Fig. 2: Effect of $f$ on the percentage of Dr for different addition concentrations at $(\mathrm{D}=0.5 \mathrm{in}, \mathrm{L}=0.5 \mathrm{~m})$
Figure 2-5 also confirmed the relation between the other variables with the $\mathrm{Dr}(\%)$ such as the addition concentration, where it can be notices that friction factor decreases by increasing the addition concentration which is also noticed with the Dr (\%) itself where it increases by increasing the concentration. The purpose behind that will be discussed in the next sections.

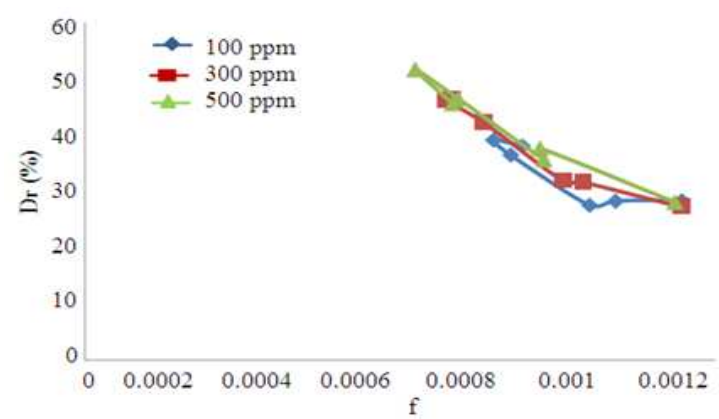

Fig. 3: Effect of $f$ on the percentage of Dr for different addition concentrations at $(\mathrm{D}=0.5 \mathrm{in}, \mathrm{L}=0.5 \mathrm{~m})$

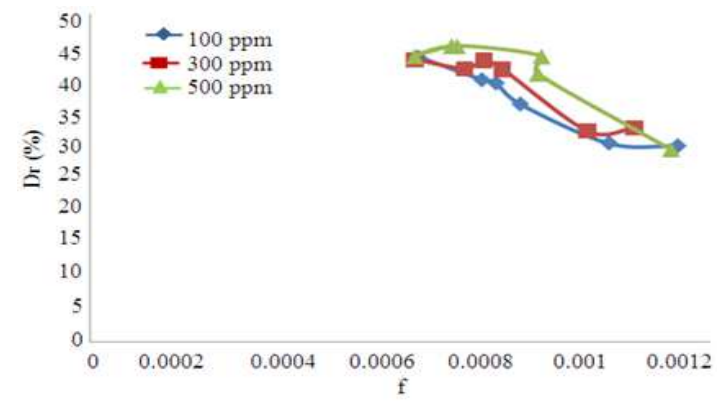

Fig. 4: Effect of $\mathrm{f}$ on the percentage of Dr for different addition concentrations at $(\mathrm{D}=0.5 \mathrm{in}, \mathrm{L}=0.5 \mathrm{~m})$

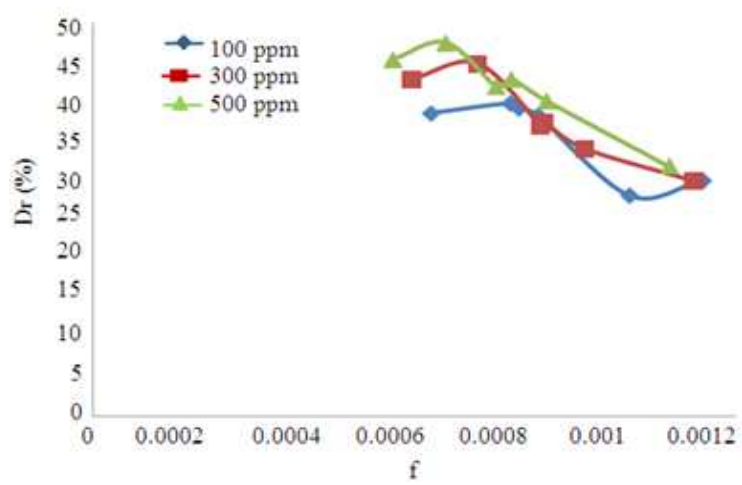

Fig. 5: Effect of $f$ on the Dr (\%) for different addition concentrations at $(\mathrm{D}=0.5 \mathrm{in}, \mathrm{L}=0.5 \mathrm{~m})$ 
Effect of Reynolds number on the percentage drag reduction: Figure 5-8, shows the effect of fluid velocity on the Dr (\%). the fluid velocity is represented by Reynolds number (Re). From Fig. 6-9 it can be noticed that the Dr (\%) increases by increasing the $\mathrm{Re}$ until certain values where the values of the $\operatorname{Dr}(\%)$ starts to be constant with the Re or it start to decline. Figure 58 , also show the effect of the addition concentration on the Dr (\%). it is clear that by increasing the additive concentration, the Dr (\%) increases.

Increasing the Reynolds number means increasing the degree of turbulence inside the pipe and that will lead us to the complicated relation between the additives and the degree of turbulence represented by Re.

Increasing the degree of turbulence means increasing the number and size of the eddies causing the turbulence which will make larger number of the additive molecules to be involved in the turbulence spectrum. That will lead to the relation between the Re, $\mathrm{C}$ and $\operatorname{Dr}(\%)$.

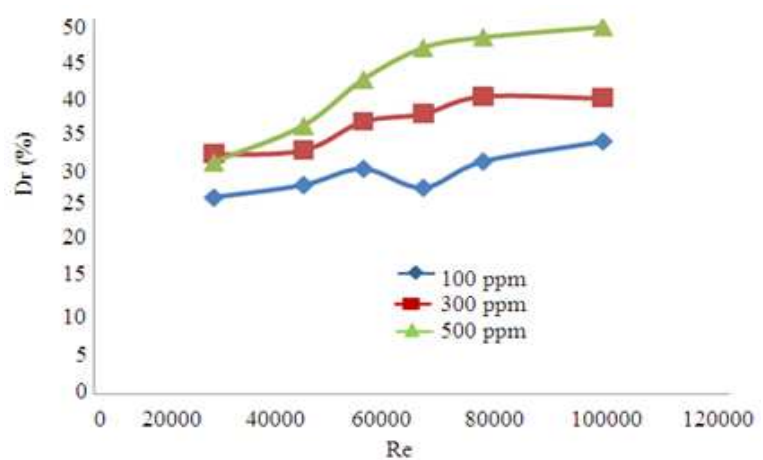

Fig. 6: Effect of $\operatorname{Re}$ on the percentage of $\mathrm{Dr}$ for different addition concentrations at $(D=0.5$ in, $\mathrm{L}=0.5 \mathrm{~m}$ )

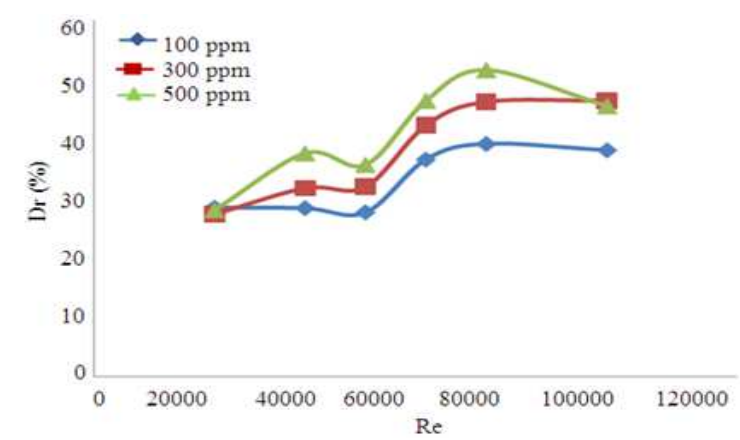

Fig. 7: Effect of $\operatorname{Re}$ on the percentage of $\mathrm{Dr}$ for different addition concentrations at $(\mathrm{D}=0.5 \mathrm{in}$, $\mathrm{L}=1 \mathrm{~m})$
Effect of addition concentration on the percentage drag reduction: Figure 9-12, shows samples of the addition concentration effect on the percentage drag reduction. Figure 10-12, shows a comparison between the in the performance of the $\operatorname{Dr}(\%)$ for different addition concentrations and the same Reynolds numbers.

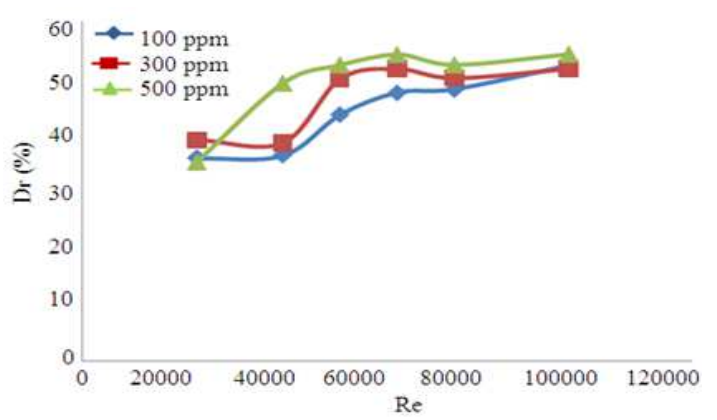

Fig. 8: Effect of $\mathrm{Re}$ on the percentage of $\mathrm{Dr}$ for different addition concentrations at $(D=0.5$ in, $\mathrm{L}=1.5 \mathrm{~m}$ )

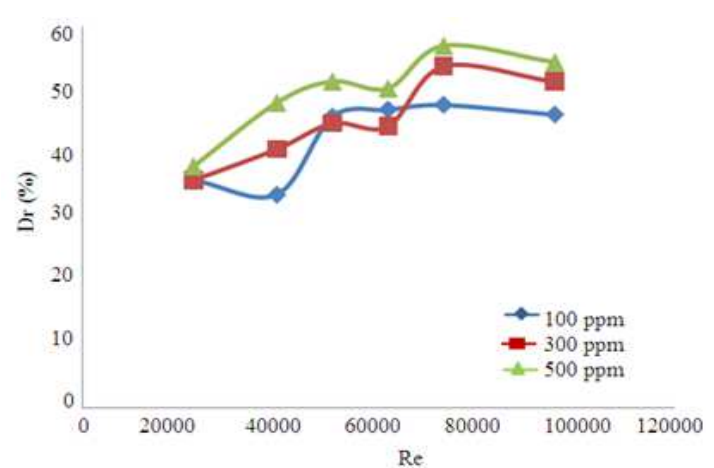

Fig. 9: Effect of $\mathrm{Re}$ on the percentage of $\mathrm{Dr}$ for different addition concentrations at $(D=0.5$ in, $\mathrm{L}=2 \mathrm{~m}$ )

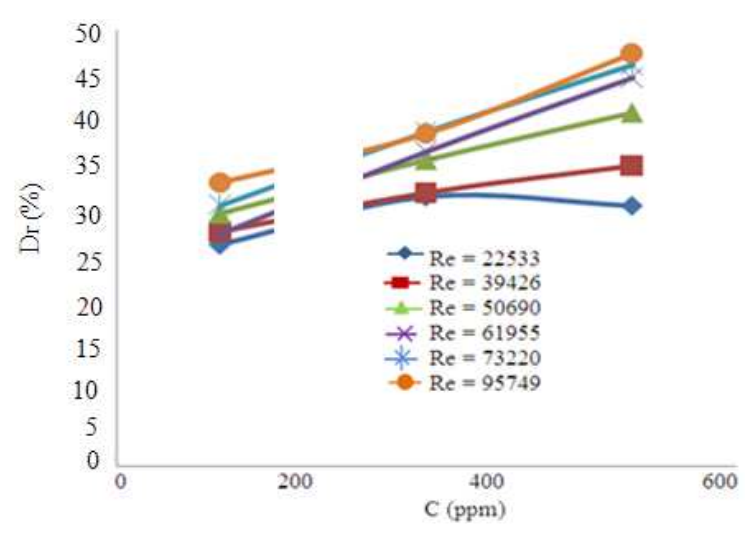

Fig. 10: Effect of adding concentration on the percentage of $\mathrm{Dr}$ for at $(\mathrm{D}=0.5 \mathrm{in}, \mathrm{L}=0.5 \mathrm{~m})$ 


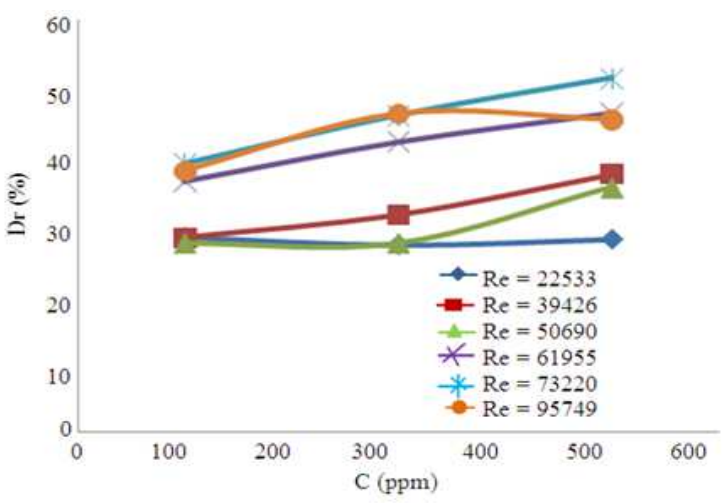

Fig. 11: Effect of adding concentration on the Dr (\%) for at $(\mathrm{D}=0.5 \mathrm{in}, \mathrm{L}=1 \mathrm{~m})$

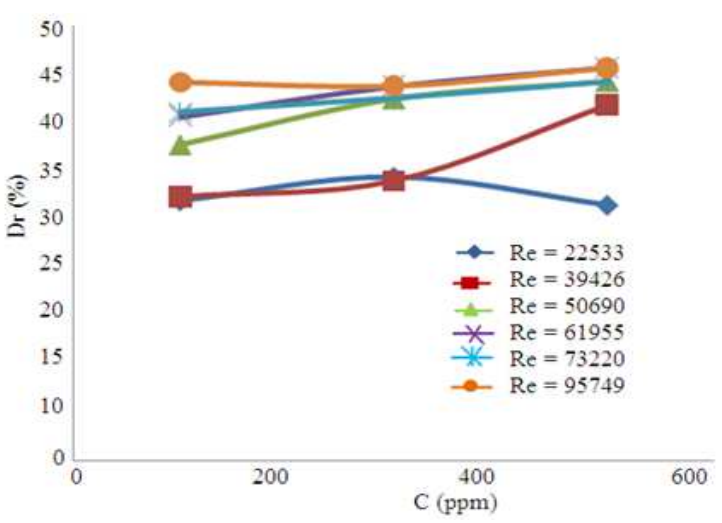

Fig. 12: Effect of adding concentration on the percentage of $\operatorname{Dr}$ for at $(D=0.5 \mathrm{in}, \mathrm{L}=1.5 \mathrm{~m})$

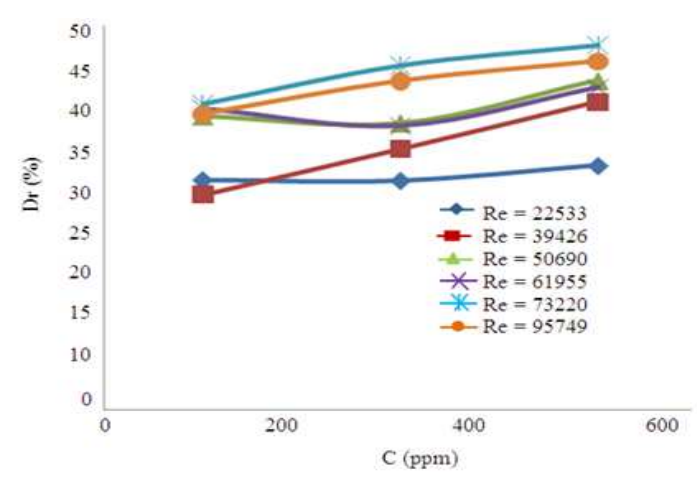

Fig. 13: Effect of adding concentration on the percentage of Dr for at $(D=0.5 \mathrm{in}, \mathrm{L}=2 \mathrm{~m})$

It is clear that the percentage drag reduction increases by increasing the addition concentration.

Increasing the addition concentration means increasing the number of additives molecules or aggregates involved in the drag reduction operation.

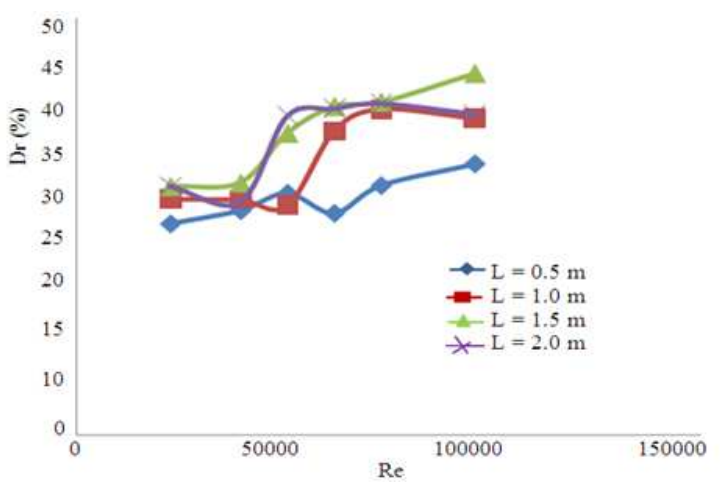

Fig. 14: Effect of pipe length on the percentage of Dr at $100 \mathrm{ppm}$ concentration

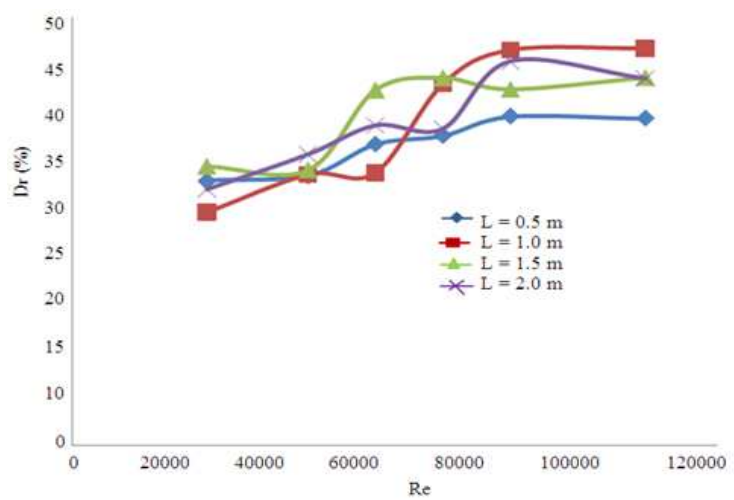

Fig. 15: Effect of pipe length on the percentage of Dr at $300 \mathrm{ppm}$ concentration

Increasing the number of molecules involved in the turbulence means increasing the turbulence area that is under the molecules effect and that will lead to the increase in the percentage drag reduction.

Effect of pipe length on the percentage drag reduction: The pipe length is one of the very interesting variables in the drag reduction science. The effect of pipe length is a clear clue on the effectiveness and the additive itself with the length and maybe the more real expression is the degree of sustainability of the additive molecules along the transportation length with all the shear stresses exposed to.

Figure 13-15 show the effect of testing section length on the percentage drag reduction for the same addition concentrations and additive type. Some of the Fig. 13-15, shows that the Dr (\%) increases by increasing the pipe length and that is not consistence with all the cases, that in some Fig. 13-15 it is not clear that the $\operatorname{Dr}(\%)$ is affected by the pipe length. 


\section{DISCUSSION}

Generally, the curves showed that the Dr (\%) increases by increasing the Re, which means increasing the additives involvement in suppressing the turbulence which will result in decreasing the pressure drop (the main indicator for the pumping power saving). The increase of the $\operatorname{Dr}(\%)$ is within certain limits of $\operatorname{Re}$ (or let say degree of turbulence) where it reaches its maximum limits of performance and after that starts to decline, the reason behind that is the relation between the degree of turbulence and the number of additive molecules in the turbulent core. Increasing the degree of turbulence will increase the number of eddy involved in the flow system and that will reduce the efficiency of the additives to cover the new spectrum generated from the increase in the turbulence.

\section{CONCLUSION}

To explain that, it is very important to understand the relation between the addition concentration, pipe length and degree of turbulence. The behavior of the Dr (\%) with the pipe length is very close to the degree of additive distribution and penetration through the turbulent media, in the present study the additive is mixed initially in the recirculation tank before the start of the operation which will ensure the best distribution of the molecules within the flow system. All that plus the mixing that happen before the entrance of the flow to the testing section. From all what mentioned before, it is clear the effect of the testing section length may be much clearer if the introduction of the additives is made by injection through the testing section.

\section{REFERENCES}

Ananthapadmanabhan, K.P., G.Z. Ma, E.D. Goddard and M. Tirrell, 1991. Surface forces measurements on a cationic polymer in the presence of an anionic surfactant. Coll. Surfaces, 61: 167-174. http://cat.inist.fr/?aModele $=$ afficheN\&cpsidt $=518$ 3401
Chapman, B., 2005. Study of drag reduction by zwitterionic and non-ionic surfactants in low temperature ethylene glycol/water recirculation systems. Ohio State University. https://kb.osu.edu/dspace/bitstream/1811/313/1/ BrianChapman.pdf

Cengel, Y.A. and J.M. Cimbala, 2006. Fluid Mechanics: Fundamentals and Applications. 1st Edn., McGraw-Hill Higher Education, USA., 0072472367, pp: 956.

Indartono, Y.S., H. Usui, H. Suzuki and Y. Komoda, 2005. Temperature and diameter effect on hydrodynamic characteristic of surfactant dragreducing flows. Korea-Aust. Rheol. J., 17: 157164.

http://infosys.korea.ac.kr/PDF/KARJ/KR17/KR174-0157.pdf

Jaghmani, I., 1999. Effect of nonionic surfactant (X$100)$ on the rheological properties of aqueous polyacrylamide solutions. M.Sc. Thesis, Concordia University Libraries.

Savines, J.G., 1964. Drag reduction characteristics of solutions of macromolecules in turbulent pipe flow. SPE J., 4: 203-214. DOI: 10.2118/867-PA

Virk, P.S., H.S. Mickley and K.A. Smith, 1970. The ultimate asymptote and mean flow structure in toms' phenomenon. J. Applied Mech., 37: 488-493. DOI: $10.1115 / 1.3408532$

Zhang, Y., J. Schmidt, Y. Talmon and J.L. Zakin, 2005. Co-solvent effects on drag reduction, rheological properties and micelle microstructures of cationic surfactants. J. Coll. Interface Sci., 286: 696-709. DOI: 10.1016/j.jcis.2005.01.055 PMID: 15897088 